\title{
Organising for Artificial Intelligence (AI) technologies
}

\section{Sukti GHOSH}

\begin{abstract}
This study focuses on organisation design choices as tools for addressing the management challenges of commercialising AI technologies for competitive advantage. It explores how design choices address fundamental problems of organising in such context, illustrating notable design features observed. Additionally, it examines external alignment and internal coherence reiterating interdependencies in organisation's design choices, when adapting to exogenous changes due to emerging AI technologies.
\end{abstract}

KEYWORDS: Artificial Intelligence, Organisation Design, Interdependencies JEL CLASSIFICATION: O3, Y4

\section{INTRODUCTION}

With rapidly rising investments in AI, globally organisations are concerned not just about ways in which AI technologies will impact their economic prospects but also ways in which investments and commercialisation of such technologies can be achieved successfully. For many organisations, this is as much a matter of making the right investment decisions as it is about optimum ways to overcome the organisational challenges of commercialising these technologies. Recent research alludes to exploring organisation design tools in overcoming challenges of pursuing emerging technologies like AI (Adner, 2017; Dong, March, Workiewicz, 2017; Kapoor \& Kleuter, 2017). Yet research on how organisation (re) design may play a role in helping organisations adopt, and progress from research \& development to commercialisation of AI technologies is scarce.

This paper argues that commercialising of AI technologies presents unique and distinct contextual factors that necessitate distinct design choices within the solution space. The study intends to make a clear distinction between AI adoption for cost minimisation purposes only (as has been common in some early adopters adopting limited AI applications for specific aspects of their non core operations) and adoption of AI technologies at scale to facilitate the firm's core business, for major product and service innovation (Bughin et al., 2017). Therefore, it aims to contribute to the existing literature in two ways: firstly, by exploring design choices observed in commercialising AI technologies for competitive advantage purposes, and secondly, exploring interdependence in organisation design (Siggelkow, 2011) through external alignment and internal coherence of design choices.

\section{LITERATURE REVIEW}

\subsection{AI Technologies Landscape}

Successful use cases of AI technologies significantly impacting organisational performance across a

The author is a graduand of the London School of Economics (postgraduate Organisational Behaviour studies). She has versatile professional experience in HR across industries including fast consumer goods, manufacturing, utility and not for profit across India and the UK. Her research interests include inter \& intra organisation design for competitive advantage. 
range of industries are aplenty. These include Amazon's \$775mn acquisition of a robotics company "Kiva" that automates picking and packing yielding a 40\% return on original investment (Bughin et al., 2017); other instances are value creation through accurate demand forecasts \& supply chain optimisation, uncovered R\&D opportunities, higher productivity, better efficiency, lower costs and enhanced personalised customer experience (Bughin et al., 2017; McKinsey, 2017). For industry purposes (as well for the purpose of this study), AI technologies constitute subfields and applications in both software and hardware based approaches of machine learning, smart robotics \& robotics automation, AI optimised hardware, computer vision and natural language processing (Brynjolfsson \& Mcafee, 2017; Bughin et al., 2017; Curran \& Rao, 2018). The impact of AI technologies on organisations is not merely realisation of efficiencies but revolutionising competitive advantages beyond what they are capable of achieving today.

Nevertheless, key organisational and management challenges in commercialising and realising opportunities presented by AI technologies (Ransbotham et al., 2017) remain. Firstly, these include the need for significant investments to benefit from these technologies. Realising true potential of AI technologies requires both resource and time investment in hardware infrastructure and software developments whether developed internally or through considered merger and acquisition, horizontal and vertical integrations to overcome restrictions on speed of AI technology deployments (Brooks, 2017; Ransbotham, Kiron, Gerbert, \& Reeves, 2017). Secondly, as AI technologies are still maturing, some in the early phases of the research-development-commercialisation cycle, there is significant disparity in understanding the applicability of these technologies (Ransbotham, et al., 2017); this is worsened by the third issue which is an acute shortage of appropriate current and future skilled talent at developer and managerial levels to help harness the potential opportunities (Plastino \& Purdy, 2018; Ransbotham, et al., 2017). Finally, experts point out that suitable data is currency for AI technology applications - unavailability of well curated structured data is likely to limit severely the organisation's capability of harnessing opportunities from AI technologies and may be a significant source of business disruption in near future (McKinsey Global Institute, 2017; McKinsey Global Institute, 2018).

\subsection{On the Problem of Organising: Design Choices and Firm Boundaries}

The problem of organising is a fundamental challenge in pursuing collaborated effort for successful fulfilment of any goal, and for profit or not. Essentially, the problem of organising may be understood as the sum of the different ways in which "labour is divided into distinct tasks and then its coordination is achieved among these tasks" (Mintzberg, 1993, p.2). Designing of organisation then is a solution search process through a problem space to address the fundamental problems associated with organising - division of labour and integration of effort (March \& Simon, 1993; Mintzberg, 1993; Puranam, Alexy and Reitzig, 2014; Puranam, 2017). Solution space mapping design choices and organisational performance are characterised by possible solution points, such that better and worse solutions exist corresponding to each (Puranam, 2017). In locating a solution point, a form of organising must thus seek to solve the four universal problems of task division, task allocation (as in division of labour) and reward provision, information provision (as in integration of effort) (Puranam et al., 2014).

Many scholars view organisation design and structure as fundamental in determining organisation's survival in the short and long term (Brickley, Smith \& Zimmerman, 2006). Early scholarly contributions discuss "strategy structure thesis" (Chandler, 1990; Galbraith, 2012; Miles et al., 1978). Later studies have led to debates on strategy following structure rather than the other way, suggesting that existing organisational structures constrain the later strategic choices the firm can make (Hall \& Saias, 1980). Scholars also suggest that structure and strategy mutually influence each other although strategy has emerged frequently to be the more important determinant of the two (Amburgey \& Dacin, 1994; Mintzberg, 1993). In recent decades, sub fields of research have emerged in line with current exogenous changes. These discuss optimality of design or the configuration approach to organisation design (Doty et al., 1993; Miles et al. 1978; Mintzberg, 1993), 
organisational contingencies such as exogenous environmental factors and technological development that drive structural changes (Puranam, Raveendran \& Knudsen, 2012), and newer organisational forms influenced by exogenous strategic changes (Puranam et al., 2014). Other work on organisation design include the managerial economics approach of instituting optimum decision rights and incentive contracts as powerful tools of design that managers can use to impact firm's performance (Athey \& Roberts, 2001, Brickley et al., 2006; Milgrom \& Roberts, 1992).

Another important aspect of organising includes decisions around firm boundaries. As firms experiment with organisational forms to survive in complex environments, decisions on activities to be integrated within the firm and non-integrated or be sourced from external markets are vital and allude to a firm's changing boundaries as part of design problems (Roberts, 2007). Coase's (1937) seminal work on firm's boundaries promulgated that a firm's boundaries are determined by the transaction costs of business activities. Furthering this thesis, scholars suggest that a different organisational form along the market-hierarchy continuum is a function of important trade-offs between internal coordination costs and external opportunity costs of value creation (Kapoor \& Lee, 2013). Additionally, decision on firm boundaries and thus design choices are motivated by other concerns such as incentive issues and difficulties in knowledge transfer, spurring a high degree of versatility in organisational forms as in networks, alliances, shared platforms as alternatives to integration of activities within firms (Holmstrom \& Roberts, 1998).

\subsection{Fitness of Design}

Scholars suggest that optimal organisation design is achieved through internal unity (internal fit) among elements of design and fitness with contextual determinants like strategy, technology, industry, markets and regulation (external fit) (Brickley et al., 2006; Miles et al., 1978; Pitts, 1980; Milgrom \& Roberts, 1992; Mintzberg, 1993; Roberts, 2007). Two theoretical propositions of significance within the concept of fit or optimality of design is configuration approach to design (Doty et al., 1993; Miles et al. 1978; Mintzberg, 1993) and complementarity of design elements (Brickley et al., 2006; Milgrom \& Roberts, 1992; Roberts, 2007). Literature suggests that the problem of organising is a complex endeavour to find solution points in the problem space to solve both, the fundamental problems of division of labour, integration of effort and problems of internal coherence \& external alignment (Miles et al., 1978; Mintzberg, 1993; Puranam et al., 2014).

Scholarly discourses propose that emergence of new technologies are exogenous aspects that require review of firm's existing optimal design choices (Brickley et al., 2006). New technologies modify division of labour, organisational coordination concerns, and fit between the organisation and its environment (Liu, Denis, Kolodny \& Stymne, 1990). For example, Thompson (1967) suggested new intensive technologies create interdependencies that need modified coordination mechanisms which in turn cause new structural forms to emerge (Barberio, 2009; Hatch \& Cunliffe, 2006), and Galbraith (2012) suggests that new disruptive technologies can create significant changes in external environment by eliminating expensive supply chains, maximising customisation and minimising economies of scale. In the context of recent emergence of AI technologies, von Krogh (2018) suggests AI as fundamental and pervasive "emerging phenomenon of economic and organisational significance" creating exogenous contexts distinct from the "average run-of-the-mill" technological innovations (von Krogh, 2018, p.404). AI technologies extend across a range of applications, present challenging pace of changes faster than organisational capabilities are able to cope with (von Krogh, 2018), and may introduce the need to adopt organisational logics that are in counter to existing ones (Kapoor \& Kleuter, 2017). Past studies propose that not only do technological revolutions by their very nature have profound impact on organisation of work, social relations, administration and manufacturing processes, but that their impact is a result of their interactions in terms of coherence and complementarity with other ancillary factors such as geography and historical era (Liu et al., 1990). Because the impact of AI technologies on organisational concerns is significantly distinct, it may be explained by the interaction effect which exerts through coherence and complementarity with other technology systems, scientific advancements and increasing complexity in various fields in the current era (Liu et al., 1990; von Krogh, 2018). Therefore, the ability of an organisation to 
respond to such emerging technological changes by adapting its form, design and boundaries may determine its ability to coordinate effective responses to changing economic and technological revolutions in high levels of uncertainty (Kapoor and Kleuter, 2017; Kapoor \& Lee, 2013) ensuring a competitive edge and it’s long term survival (Brickley et al., 2006).

Although several scholarly reviews examine optimality of organisation design choices, there remains a visible gap, where effective organising for commercialising AI technologies are concerned. This paper argues that AI technologies present unique, distinct set of contextual factors that may necessitate distinct design choices within the solution space. The specific research objectives can therefore be summarised as:

(a) To explore organisation design choices observed in firms commercialising AI technologies for competitive advantage (b) To analyse observed design choices for internal fit or interdependency (c) To analyse observed design choices for external alignment with the context of AI technologies.

\section{METHODOLOGY}

An exploratory qualitative research for theory-building inductive approach was pursued. An in-depth embedded single case based research design was adopted and facilitated by qualitative data collection methods (semi structured interviews triangulated with data from multiple sources). Data analysis was conducted using analytic induction strategy and content analysis methods.

\subsection{Methodology Considerations}

In light of the lack of academic work exploring organisation design choices in commercialising AI technologies, an exploratory study was deemed appropriate, with theory-building inductive approach as desired outcome of the research pursuit. Eisenhardt \& Graebner (2007) comment that research questions that are of crucial significance for organisations and/or theory and exhibit that existing research do not address the research question adequately is justified for a theory building exploratory research. An exploration methodological approach is also suitable when a certain theoretical area has grown to maturity along certain known continuum (e.g. organisation design literature), yet significant changes have occurred along the way (e.g. context of AI technologies) justifying it to be explored afresh (Stebbins, 2001). Although exploratory approach can be both quantitative or qualitative, in this case a qualitative strategy was chosen for in-depth study of observed design choices to gain greater benefit from a rich set of detailed data, systemic and contextual understanding (Patton, 2002).

Exploratory qualitative research can be implemented through various research designs, but in this instance a case study was deemed as most suitable and reasonably so as Yin (as cited by Ridder, 2012, p.93) suggests that case studies are highly effective in understanding complex phenomenon, contemporary in nature such as organisational processes within its context, specifically when the boundaries between the phenomenon and the context may not be clear. The phenomenon of commercialising AI technologies in organisations and design choices made in such a context is both contemporary and within a real-life context. Due to the study being conducted in a single frame in time, without advantages of longitudinal observations, the boundaries between the context and design choices (both in temporal and spatial terms) are not clear (Yin, 2012). Additionally, for this study, although the unit of analysis is the organisation, other units of analysis emerged at division and team level, and therefore the research design evolved into embedded single-case study design (Yin, 2012).

However, case study design has often been plagued with accusations of being non-scientific due to its lack of generalizability and given the specificity of context it demonstrates for the case study unit (Dubois \& Gadde, 2002). Yet over the years with progressing academic debate, this very feature of case studies, the interaction between phenomenon and its context for informed learning and deeper understanding from single cases has become its strength rather than weakness (Flyvbjerg, 2006); in 
fact scholars agree that advancing scientific knowledge has as much to gain from in-depth understanding as from statistically supported generalizable results (Dubois \& Gadde, 2002; Flyvbjerg, 2006).

\subsection{Case Organisation}

Validity concerns in case study research can be put to bed when strategic choices are made in sample selection. Flyvbjerg (2006) suggests pertinent strategies for sample case selection - an information oriented selection strategy as apt for selecting cases on the basis of their information content to maximise the utility of information from small sample numbers.

An initial review of publicly available data on organisational cases reported in major business \& management analysis sources like FT, Harvard Business Review, MIT review, Forbes and Bloomberg led to shortlisting of potential case samples. Featured articles or analysis were reviewed for specific organisational cases highlighting examples of systematic AI technology commercialised for core businesses and competitive advantage. The potential case shortlists represented automobile, technology and retail sector firms and contacts were sought through common networks to approach these organisations. At least 3 organisations were approached and requests made to discuss purpose and scope of study with relevant member of management team. Unfortunately, two organisations expressed some concerns about sharing organisational data around AI technology projects as these were considered too strategic, secretive or nascent for feasible data sharing thus leaving the third organisation to be selected as the case sample facilitated by feasibility of and access to information. The selected case sample, a UK based Internet retail player and technology platform solutions provider, was approached through common professional contacts. The case organisation a member of FTSE 100 index, is referred to as Z2W PLC for purposes of this study. Z2W was established in 2000 and has approximately 11,900 employees in the group (2016) of which about 1300 employees are in its technology division representing 45 different nationalities. Although Z2W initially forayed as an online only retailer over the years it has evolved to become a platform business providing technology solutions to UK and international clients, evolving into a dual retailer and technology solutions provider. Suitable evidence of commercialised AI technologies for competitive purposes were found both from examples discussed in business analysis reports, their website, and also initial scoping discussions with the management team.

\subsection{Data Collection and Analysis Strategy}

As highlighted previously, case study research is often riddled with concerns regarding validity (construct, internal \& external) and reliability (Yin, 2012). Yin (2014) suggests using multiple sources of evidence, creating a case study database, maintaining a chain of evidence and exercising care when using electronic sources for establishing construct validity and reliability of evidence. Multiple data sources were used in constructing evidence database to allay these concerns. Additionally, careful curating of reliable and up to date electronic information and seeking data in unusual places like organisational technology blogs, annual reports and other publications on firm's website (Puranam, 2012) ensured effective data triangulation and strengthening of construct validity (Dubois \& Gadde, 2002; Yin, 2014). The qualitative data collection strategy was executed through semi structured open-ended interviews, review of documents (newspaper articles, industry expert analysis), archival records (committee discussions, firm's blog site, website data, organisational reports) and physical artefacts (company presentations, demos, virtual tours of technology solutions). Data from various sources was collected between March - June 2018.

An interview guide was developed for semi-structured interviews. An initial review of literature and organisational case reports were used to draft appropriate questions. A case study protocol identified the areas of enquiry as: AI technologies commercialised and firm's objective of doing so, what organisation design choices facilitated this and how, challenges/opportunities faced and contextual factors in commercialization and design choices. A total of 15 participants distributed between 
employee and management hierarchies from different departments were interviewed and each interview lasted an average 55 minutes. Additionally, spending time with the data and going back and forth between literature and data collection (Dubois \& Gadde, 2002) during interviews facilitated effective utilisation of data collection tools. A particular unavoidable shortcoming in data collection was the restricted timelines, however focused review of archival data was used to access relevant information and contextualisation of organisation's past and present events.

An analytic induction strategy for data analysis was employed which involves quasi-deductive approach followed by inductive approach (Patton, 2002). Additionally, as during data collection, adopting a conscious strategy of examining plausible rival explanations helped reduce validity and reliability concerns (Yin, 2014). Analytical techniques included discovering patterns, themes and categories generated by the researcher interacting with the data via content and thematic analysis (Patton, 2002). Based on these techniques, data from interview transcripts and other sources was manually analysed and organised into 1st order codes and 2nd order themes (Gioia, Corley \& Hamilton, 2013). First (1st) order depicts interaction with rich descriptive data like open coding adhering to participant terms, distilling to numerous first order codes, albeit subjective (Gioia et al., 2013), yielding a mind boggling 36 codes. These codes were then judged for internal homogeneity and external heterogeneity (Patton, 2002) and 15 basic codes were retained after multiple rounds of analysis. Second (2nd) order analysis was more theoretically oriented and examined first order findings for underlying interpretations that attend to nascent concepts with adequate referents in literature to discern deeper understanding not perceptible to organisational members (Gioia et al., 2013; Hope, 2010). This yielded 7 second order themes and 3 aggregate dimensions that formed the basis of a data structure. A data structure (see appendix II) represents visually the first order codes, second order themes and aggregate dimensions to indicate progress from raw data to themes in analysis and thus rigor in qualitative analysis (Eisenhardt \& Graebner, 2007; Gioia et al., 2013).

\section{FINDINGS}

\subsection{AI Technologies: Commercialising for Competitive Advantage}

Analysis indicated significant commercialisation of AI technologies in $\mathrm{Z2W}$ for achieving competitive advantage; also pervasive commercialisation of AI technologies in strategic and operational projects with competitive advantage gained through predictive analytics, managing complexity, real-time optimisation, forecasting, personalisation of customer experiences and embedding AI to produce commercially superior technology solutions for platform business clients. Participants cited multiple examples of AI technology use cases and discussed commercial advantages.

"..the robots are an obvious example....we are using deep learning to detect frauds, recommendations on websites, we are trying to predict what you're going to buy next, all of your shopping list, even the whole routing algorithms....predicting what stock ....." "I do think AI in general gives the competitive advantage to companies because it makes your experience much better.... All the services that we provide to customers and even our very low purge results that came out because of our AI, machine learning solutions ......" (P11)

"..(AI) it's a massive area for us ...artificial intelligence and to a large extent robotics pervades our e-commerce fulfilment of logistics platform, they crop up everywhere ... we use AI machine learning at the front end to help if you like customers shop faster with less friction.." (D12)

Commercialising AI technologies for competitive advantage seems to present unique challenges and thus contextual strategic imperatives. Three unique strategic imperatives associated with AI were identified on analysis: appropriate management and governance of data, intersectional or multidisciplinary thinking and applications in implementing AI technologies and future proofing orientation to keep ahead of emerging competitive threats. These three distinct imperatives are 
summarised as: data governance (Teachey, n.d.), intersectional approach and future proofing. Participants commented on these as:

Data Governance: "So probably beyond coming up with a bright idea how to apply some technique I've seen outside or indeed techniques developed outside the state-of-the-art, the biggest thing that seems to be coming up all the time is data. We are generally quite fortunately positioned so years and decades of data from warehouses from every aspect monitored, logged .... " (P2)

Intersectional approach: "So we have one sub team that specialises in machine learning and Intelligence we have got some teams that are particularly good at hardware and electronic prototyping we have got some other sub teams around algorithms and deeper thinking. There is a lot of interdisciplinary thinking, we have people in our teams who are multidisciplinary themselves.. "

"So with disruption and Innovation the sharing of ideas is really critical if somebody has a really great idea but doesn't talk to other people or does and others don't listen then we're missing out on opportunities so it's one of the key things that we might want to focus on.. " (P7)

Future proofing: "..... one threat is simply that the way people shop is innovating all the time and so we need to make sure that our business model doesn't become out-dated and is refreshed all the time....so it's about staying ahead in terms of robotics and automation and making sure that we are looking for opportunities to use it and also we always have strong skills in that areas if you don't have the skills you won't even perceive those as a threat because you don't occupy that space." (P3)

"Strategically it also wants to stay ahead of the curve and not allow has to be kind of swallowed up or overtaken by other companies...I think there's definitely a view people such as our CTO and widely within the industry that if you don't disrupt yourself you'll be disrupted by other people so that's always in people minds so I think that kind of drives what we do." (P9)

\subsection{Organisation Design}

\subsubsection{Firm's Boundaries}

Interesting findings emerged on firm's decision to integrate (in-house) or non-integrate (outsource) activities thus defining firm's boundaries. Although Z2W initially began as an online retailer, it had historically adopted a stance of developing all software and more recently developing AI technology applications in-house. This decision seemed to be influenced by three factors - an inability to find software systems appropriate for its purpose necessitating large investments in resources and time to rework software purchased from external vendors (transaction costs), it created uncertainties around the success of its novel business model (adaptation) and concerns around having control of its software systems \& data and hence capability in the long run (knowledge concerns). Participants explained:

Transaction costs: "....this system (vendor's software) could only give us something like 14 drops and we wanted more but it couldn't do more because it wasn't designed for our problem ....so we developed the capability internally to write our own software we hired some computer scientists and mathematicians with PhDs in the topic and wrote our own systems and these days the number of drops have increased significantly" (P3)

Adaptation: "So it's definitely the preferred option to build things in house ........we are particularly approaching a problem that other people haven't figured out yet so the little corner of logistics that we operate in is quite extreme in terms of the complexity of the operations so there are no off the shelf solutions to it...." (P15)

Knowledge concerns: “..that came from both realisation that we wanted to have control of our own destiny in terms of what our software could do and if you are using other people's software because 
we are in a unique industry nobody else has our business model we are then asking third parties to tailor their packages but other package wasn't even that designed for our industry so we are better off trying to design our own." (P3)

\subsubsection{Division of Labour}

Three important organisational goals for Z2W were identified: growing and sustaining the UK retail business (commercialisation), developing the smart platform technology business (research \& development leading to commercialisation) and engage in continuous disruptive innovation to thwart competitive threats for firm's sustainable future (future proofing).

"...also challenge Z2W retail with different ideas....Are there areas related to that where we might want to augment our current function. I think that's important for a second purpose for if we can show that we are doing things well and the UK retail side, then maybe we'll have a better chance of selling our technology as a platform to the international retailers. I think the goal number 2 from my perspective is to be able to offer a platform to international retailers and leverage our technology and our IP in that way. The third thing we might try to do also in order to both of those streams as well as other subsidiaries that may come out innovate around that core business." (P2)

"...I think more recently that has shifted towards selling a platform to the world so rather than licensing a technology to provide a platform for other grocery retailers so that's one the obvious core goals of Z2W strategically I think. Strategically it also wants to stay ahead of the curve and not allow to be kind of swallowed up or overtaken by other companies." (P9)

On analysis these goals were mapped into three major tasks of research and development (R\&D), commercialisation and future proofing Z2W. Since, Z2W had integrated activities pertaining to software development and thus R\&D of technologies, participants believed that growth of its technology division and internationalisation to build organisational capability was a result of this decision. Participants noted that teams either had research or commercialisation focus (some integrating both depending on their position in the value chain). Additionally, structural reorganisation had enabled the organisation to focus distinctly on future proofing through disruptive innovation \& external strategic partnerships.

$\boldsymbol{R} \& D$ : "We have put a lot more emphasis on research, we have not a division but a loose grouping of all the research teams now - $X$, robotics, simulation, us (data science) and machine learning services that are part of $<$ Smart Technology Platform $>$ " (P14)

Commercialisation: "....more recently we have created Solutions division to deliver the Z2W smart platform to companies around the world ......" (P12)

Future Proofing: "So I run a team of 20 people and that team is called the X team and the concept of $X$ is that if you're trying to make a radical change or disruptive improvement or trying to improve something by 10 times then you might think differently about how you go about doing that than if you were to make something for example a little bit better." (P1)

\subsubsection{Integration of Effort}

Analysis yielded two important design features in integration of effort - providing clear non conflicting focus to teams \& individuals, incentivising self-development and collaboration (incentive provision) and multiple tools for collaboration, knowledge exchange (information provision). Additionally, standards \& protocols helped reduce need for explicit information exchange facilitating integration.

Incentive provision: "It's (the new careers model) trying to be more focused on a broader set of skills than previously we had a tendency to only promote and assess based on technical ability but we are incorporating things like collaboration, trust, the ability to learn quickly, autonomy and also the ability to sort of help other people improve as well. So it's trying to enhance our value of the softer 
skills so collaboration for example so previously we promoted based on purely technical ability whereas now we are saying that you might be technically excellent but if they can't collaborate very well we are not going to reward them for that because we want to have people that can work well together so that if we have more collaborative environment and feed off each other's ideas.." (P7)

Information provision: "...we now have essentially a community of practice so there's a machine intelligence practice .... They essentially look at initiatives for people of I supposed to have similar interests how do we share knowledge there. They try to bring together all these disparate groups of people into more of this kind of collective it's like a community type thing..." (P4)

"So slack is very good, communication is very important because it's asynchronous and people can catch up with what's going on with most projects have their own slack channels and even if it is just one person working in the project, we encourage them to write down results, report them on slack channel... " (P10)

\subsection{Interdependence}

\subsubsection{External Alignment}

Analysis revealed external alignment of design features with exogenous contextual variables and thus with the three unique strategic imperatives of commercialising AI technologies - data governance, intersectional thinking and future proofing, which seemed to prompt adaptation in design features for effective organisational response. Evidence indicated, organisational design changes such as investing in resources (tools like Cloud \& BigQuery, embedded data science teams) to improve availability, storage and use of data; building organisational capability along multiple disciplines, multidisciplinary teams and fostering internal and external alliances to encourage intersectional approach; formation of group CTO role, innovation units like X team and actively pursuing external alliances to ensure future proofing of $\mathrm{Z2W}$.

Data Governance: "We use a lot of Google Cloud and that has been a massive liberator for us things like BigQuery that's been revolutionary for us ... now we basically push everything onto BigQuery and each and every data scientist works off the same set of data but without any of the kind of contention" (P4)

"We have been trying to evangelize the data and data practices with these teams both with development teams because they generate data and with analyst teams from retail and business planning so it's quite nice for the relationship to be better." (P11)

Intersectional Approach: "So we are a Multidisciplinary team so we have got mathematicians, physicists and computer scientists, electronics engineers and mechanical engineers so that we can cover a breadth of innovation and within the computer science discipline we have got people who specialise in machine learning and artificial intelligence." (P1)

Future Proofing: I now run the office of what's called Z2W's CTO and my remit is to do everything that is not looking after building existing platforms, so it's research, it's advanced research, it's our relationship with the government which is growing more complex by the day, it's our relationship with universities and schools our <outreach program> which is our educational program and it's our technology Brand and how we project that and build our technology brand which is important in terms of hiring and retaining the talent we have, it's about future proofing $\mathrm{Z} 2 \mathrm{~W}$, it's about intellectual property strategy and then it's about things that we don't talk yet very actively - it's about technology spin outs.." (P12) 


\subsubsection{Complementarities}

Complementarities in design choices were observed in multiple instances. An interesting finding was that informal design features such as organisational culture \& values complemented other design features like changes in task division or integration of effort (information provision) in response to changing external context.

"..we built a huge talent pool which means that we are able to tackle a huge range of problem even if the skills are not within one team its having them within the same organisation so it's having the talent pool......we consciously acquire that talent pool, there is a problem we want to solve and the preference has always been to solve the problem internally ...if you haven't got the right skills you need to, you need to find where to get them into the organisation ..." (P3)

"So it's trying to enhance our value of the softer skills so collaboration for example so previously we promoted based on purely technical ability whereas now we are saying that you might be technically excellent but if they can't collaborate very well we are not going to reward them for that because we want to have people that can work well together so that if we have more collaborative environment and feed off each other's ideas.. " (P7)

\subsection{Rival Explanations}

An alternative perspective that emerges from the evidence providing support for factors other than AI technologies for organisation design choices is signalling effect. Signalling here refers to organisational activities undertaken with an aim of conveying to the market and stakeholder's certain information that it wants to highlight about itself to project a favourable image (Bangerter et al., 2012). Some participants reflected that it was important that the organisation is viewed externally as being ground breaking and innovative and that the brand is projected as a progressive technology firm. This is helpful not only to attract potential clients for its smart technology platform but also to attract and retain valuable skilled talent in a highly competitive market. Some design features like the growth of technology division, $\mathrm{X}$ team, external alliances seem to be associated with this perspective.

"I think main reason why people are attracted to us from that perspective besides the fact that it is a whole package is that they see us as a ground breaking and innovative." (P2)

"..so there was a big cultural change I would say and quite a big branding drive to create $\mathrm{Z} 2 \mathrm{~W}$ technology as a thing and that was mentioned in the same breath as companies like Google ...." (P10) "...by being able to say we are leveraging the latest master technology in order to achieve these things that make our product more competitive product and so I think it's a big part of our brand in the sense that we use these technologies and therefore our product is more appealing." (P13)

\section{DISCUSSION}

AI technologies are a major source of change in organisation's exogenous context and demand effective response for on-going success and eventual survival. This case study explores the organisation design features of a UK based firm (retail and technology business) commercialising AI technologies for competitive advantage. Findings indicate that commercialisation of AI technologies present at least three unique strategic imperatives for the organisation namely data governance, intersectional approach and future proofing. The study discusses organisation design choices as potential tools for addressing adaptation challenges in commercialising AI technologies for competitive advantage. Design choices include decisions regarding boundaries and responses to fundamental problems of organising (task division, task allocation, incentive and information provision) (Puranam et al., 2014). In addition, organisational adaptation to strategic imperatives and 
thus exogenous context is examined through external alignment in design features. The examination of fit demonstrates that along with external alignment to strategic imperatives, organisation design features also exhibit complementarity such that changes in a dimension of design prompts changes in another complementary design choice (Puranam et al., 2014). Thus, it reiterates the concept of interdependencies (Siggelkow, 2011) in organisation design choices. A conceptual framework is illustrated in appendix III.

Along with the three strategic imperatives, the evidence helped identify Z2W's three distinct organisational goals namely $\mathrm{R} \& \mathrm{D}$, commercialisation and future proofing. The data governance imperative necessitates organisational systems, processes and activities for availability, curating and integration of high quality data in sound business decision-making (Wise, 2016). It steps beyond data management and highlights that data of key importance (McKinsey Global Institute, 2018) is the first step in integrating AI technologies with business. Such integration needs to be widespread, not just restricted to specific management or data science organisational corners and begs a cultural shift (Wise, 2016). Z2W's design response to this imperative span beyond investing in technological tools like BigQuery for effective data storage \& access. It included formal design choices of task division and allocation such as increasing the size and number of data science teams for research and applied research, embedding data scientists in production teams to ensure close feedback to encourage appropriate data curating as well as to enable production teams to apply AI technologies in production activities. This helped to close the research to production gap, enhancing successful commercialisation (Kapoor \& Kleuter, 2015), exhibiting complementarities between decision to integrate R\&D activities for successful commercialisation and team structure. Informal design tools such as informal interaction (special interest groups, internal conferences) between data science and production teams leading to data democratisation and evangelising of data mind-set influenced cultural shift \& integration of AI technology applications in business decisions. Informal elements like culture play an important role in reinforcing formal design decisions (Defillippi et al., 2009; Siggelkow, 2011).

The intersectional imperative is approach combining multiple disciplines in problem solving and decision-making. Z2W's design response to intersectional approach involved among other things encouraging collaborations internally (multidisciplinary teams, building organisational talent across variety of disciplines, formal and informal tools for collaborations) and externally (collaborations with universities, research, technology firms, active technology internship program). Complementarities in design choices included - encouraging organisational members to engage in collaborative thinking with opportunities and tools for collaboration (information provision) while also incentivising (incentive provision through pay and promotions) collaboration as an organisational value through the careers model (Roberts, 2007). Notably, promoting collaboration using informal design choices such as culture (catalyst \& agile coaches) to bolster the intersectional approach (Brickley et al., 2006; Defillippi et al., 2009; Rivkin \& Siggelkow, 2003).

The future proofing imperative is shaping the organisation to develop continued resilience against future uncertainties and emerging competitive threats. Z2W's response to this imperative begins with future proofing as one of the three organisational goals. Design choices include developing and growing research and innovation teams like $\mathrm{X}$ team (disruptive innovation unit), creating the group CTO role and developing range of relationships externally with suppliers, technology partners, research firms, universities, government \& policy making bodies. Scholars suggest that organisations adopt a range of methods - in-house development to extensive research partnerships, to overcome barriers in traversing from R\&D to commercialisation of radical new technologies (Kapoor \& Kleuter, 
2015). Integration of technology development (vertical integration of $R \& D$ in this case) enables firms to commercialise new technologies faster (Kapoor \& Adner, 2012). Therefore, establishing distinct $X$ team as an innovation unit helped exploration of potential new technology ventures (Isherwood \& Tassabehji, 2016) as well served as dedicated boundary-spanning unit scouting external environment for new opportunities and diffusing such knowledge within the organisation (Kleuter \& Monteiro, 2017). To that end, the group СTO role also plays important boundary-spanning functions by establishing and maintaining extensive relationships in the external environment and creating avenues for integration of such knowledge internally (Kleuter \& Monteiro, 2017). The creation of group CTO role may serve other purposes. Scholars have suggested that firms are deeply embedded in an ecosystem characterized by interdependent activities of its suppliers, customers and complementors (e.g. government, schools, and universities) (Kapoor \& Lee, 2013). The adaptability of a firm in modifying its organisational form to influence these interdependent activities in its ecosystem (through CTO activities) enhances successful commercialisation of radical technologies in rapidly changing exogenous contexts (Kapoor \& Lee, 2013). Additionally, in such exogenous contexts, increased information processing is vital for organisation's survival, achieved by increasing management bandwidth (e.g. Monsanto's "two-in-a-box" structures) to focus on critical aspects (Galbraith, 2012); the CTO office role seems apt for the purpose. A firm's alignment with its ecosystem structure and thus boundaries may also fulfil a focal customer value proposition (Adner, 2017) such as Z2W's integration with Alexa voice services as part of a larger AI ecosystem of seamless customer services which eventually may become source of competitive advantage.

Z2W's decisions on integration and non-integration of business activities thus boundaries indicate factors beyond transaction costs such as concerns around knowledge transfer, adaptation to uncertainty (Gibbons, 2005; Holmstrom \& Roberts, 1998). Additionally, organisations pursue a range of boundary options including vertical integration, strategic alliances with key players, quasi-boundary forms with external bodies to respond to uncertainties due to new technology changes (Luke, Begun \& Pointer, 1989; Tushman, Lakhani \& Lifshitz-Assaf, 2012) by influencing interdependent activities of players in its ecosystem (Adner, 2017; Kapoor \& Lee, 2013). Z2W's task division decision of integrating $\mathrm{R} \& \mathrm{D}$ activities is complemented by growing a separate technology division. It facilitates building large talent base and organisational capability in different disciplines to complement future proofing activities and intersectional thinking for commercialising existing and unexplored competitive terrains (Kapoor \& Adner, 2012). Additional notable features of Z2W's structure include the multidivisional form and the integration of product organisation complete with chief product officer, product owners and task allocation based on product missions. These structural elements facilitate decentralisation in decision rights \& incentives (Brickley et al., 2006) and also enable organisational members to focus on products facilitating R\&D to commercialisation of AI technologies (Kapoor \& Adner, 2012; Marengo \& Pasquali, 2012).

Overall then, Z2W's design choices illustrate two practical implications. One, that managers may use design tools to shape better decision making (Siggelkow, 2011) such as data driven organisational decision making, foster collaboration or avoid multitasking dilemmas (Brickley et al., 2006). Two, that informal design tools like organisation culture $\&$ values when selected carefully complement and reinforce formal design choices (Brickley et al., 2006; Defillippi et al., 2009; Rivkin \& Siggelkow, 2003). The rival perspective suggests some adopted design changes may have been motivated by the firm's desire to signal to clients and prospective talent a positive brand that is ground breaking, innovative and comparable to organisations like Google. Signalling theory explains that in situations of information asymmetry, one party may convey useful signals to the other to reduce such asymmetries (Bangerter et al., 2012). It is however unlikely that signalling alone could have driven so 
many structural aspects. For example, if attracting and retaining highly skilled talent is desired outcome of such signalling, other on-going design choices such as providing challenging work (e.g. research oriented projects), collaborative work environment (e.g. tools \& forums to collaborate), clear career goals (the careers model with specific foci) and incentives to innovate (e.g. monetary incentives on successful patents, career progress based on technical expertise) will be necessary to produce lasting impact on talent retention. In short, the rival perspective alone may not have led to observed design choices and interdependencies require that design choices reinforce each other (Siggelkow, 2011) creating internally coherent \& externally aligned design.

\section{LIMITATIONS AND FUTURE RESEARCH}

This case study investigates the concern of commercialising AI technologies for competitive advantage and explores tools of organisation design to address these problems from management perspective. It demonstrates external alignment and internal coherence of design choices. Importantly, it attempts to show that prompted by changes in exogenous context, transformations in the solution space for organising will display changes in one problem dimension when a complementary solution is found in another problem dimension exhibiting complementarity (Puranam et al., 2014). Thus, reiterating an organisation as a system of interdependent choices (Siggelkow, 2011) and that external alignment and complementarities are core to success of organisation (re) design endeavours. The focus on interdependencies enhances perspectives on design choices to survive and compete in uncertain environments of rapid technological developments. Both these concerns have not been explored previously in the context of commercialising AI technologies for competitive advantage. Additionally, it introduces three key strategic imperatives, managers may focus on to successfully commercialise AI technologies for competitive advantage. Also, it provides empirical evidence that may be useful for greenfield and brownfield endeavours in adapting organisation design efforts for successful AI commercialisation.

However, important limitations remain. Firstly, the limited time frame within which the study was conducted. Although some access to historical documents was available, they do not compensate for a longitudinal study and place severe limitations on establishing any causal relationships between the context of AI technology commercialisation and organisation design choices made. It is difficult to comment with confidence on the observed strategy structure relationship (Chandler, 1990) as well as on the direction of the observed effect in this case. In absence of a longitudinal study it cannot be stated with confidence whether strategic imperatives alone were responsible for observed structure or if existing structural elements impacted both strategic imperatives and evolving design features (Kapoor \& Lee, 2013; Mintzberg, 1993; Siggelkow, 2011).

Potential for further research remains in longitudinal and confirmatory studies concatenating to the findings here to infer results that are generalizable and contribute in other respects (Stebbins, 2001) such as direction of effect or impact of existing structural elements on adoption of redesign choices. Additionally, other interesting enquiries include how commercialised AI technologies may lead to increased ecosystems as structures (Adner, 2017) for competitive prerogatives.

\section{REFERENCES}

Adner, R. (2017). Ecosystem as structure: an actionable construct for strategy. Journal of Management, 43(1), 39-58. DOI: $10.1177 / 0149206316678451$ 
Amburgey, T. L., \& Dacin, T. (1994). As the left foot follows the right? The dynamics of strategic and structural change. Academy of Management Journal, 37(6), 1427-1452. DOI: 10.5465/256794

Athey, S., \& Roberts, J. (2001). Organizational design: Decision rights and incentive contracts. American Economic Review, 91(2), 200-205. DOI: 10.1257/aer.91.2.200

Bangerter, A., Roulin, N., \& König, C. J. (2012). Personnel selection as a signaling game. Journal of Applied Psychology, 97(4), 719-738. DOI: 10.1037/a0026078

Barberio, V. A. (2009). Evolutionary dynamics of coordination-communication networks in open source development (Doctoral dissertation, alma).

Brickley, J. A., Smith Jr, C. W., \& Zimmerman, J. L. (2006) Managerial Economics and Organizational Architecture, (3rd ed.). McGraw Hill.

Brooks, R. (2017). The Seven Deadly Sins of AI Predictions. MIT Technology Review. Retrieved from https://www.technologyreview.com/s/609048/the-seven-deadly-sins-of-ai-predictions/. [Accessed 2018-07-20].

Brynjolfsson, E., \& Mcafee, A. (2017). The business of artificial intelligence. Harvard Business Review. Retrieved from

https://starlab-alliance.com/wp-content/uploads/2017/09/The-Business-of-Artificial-Intelligence. pdf. [Accessed 2018-07-20].

Bughin, J., Hazan, E., Ramaswamy, S., Chui, M., Allas, T., Dahlström, P., Henke, N., \& Trench, M. (2017). Artificial intelligence-the next digital frontier. McKinsey Global Institute. Retrieved from https://www.mckinsey.com/ /media/McKinsey/Industries/Advanced\%20Electronics/Our\%20Insi ghts/How\%20artificial\%20intelligence\%20can\%20deliver\%20real\%20value\%20to\%20companie s/MGI-Artificial-Intelligence-Discussion-paper.ashx. [Accessed 2018-07-20].

Chandler, A. D. (1990). Strategy and structure: Chapters in the history of the industrial Enterprise, 19. pr. (Vol. 120). MIT press.

Coase, R. H. (1937). The nature of the firm. Economica, New Series, 4(16), 386-405. DOI: $10.2307 / 2626876$

Curran C. \& Rao A. (2018). Briefing: Artificial Intelligence. PwC report. Retrieved from http://usblogs.pwc.com/emerging-technology/briefing-ai/. [Accessed 2018-07-18].

Defillippi, R., Arthur, M., \& Lindsay, V. (2009). Knowledge at work: Creative collaboration in the global economy. John Wiley \& Sons. pp.75-100.

Dong, J., March, J. G., \& Workiewicz, M. (2017). On organizing: an interview with James G. March. Journal of Organization Design, 6(1), 14. DOI:10.1186/s41469-017-0024-z

Doty, D. H., Glick, W. H., \& Huber, G. P. (1993). Fit, equifinality, and organizational effectiveness: A test of two configurational theories. Academy of Management journal, 36(6), 1196-1250. DOI: $10.2307 / 256810$

Dubois, A., \& Gadde, L. E. (2002). Systematic combining: an abductive approach to case research. Journal of business research, 55(7), 553-560. DOI: 10.1016/S0148-2963(00)00195-8

Eisenhardt, K. M., \& Graebner, M. E. (2007). Theory building from cases: Opportunities and challenges. The Academy of Management Journal, 50(1), 25-32. DOI: 10.5465/amj.2007.24160888 


\section{Japan Social Innovation Journal, Vol. 8, No. 1, 2019}

Flyvbjerg, B. (2006). Five misunderstandings about case-study research. Qualitative inquiry, 12(2), 219-245. DOI: $10.1177 / 1077800405284363$

Galbraith, R., J. (2012). The Future of Organization Design. Journal of Organization Design, 1(1), 3-4. DOI: $10.7146 /$ jod.6333

Gibbons, R. (2005). Four formal (izable) theories of the firm?. Journal of Economic Behavior \& Organization, 58(2), 200-245. DOI: 10.1016/j.jebo.2004.09.010

Gioia, D. A., Corley, K. G., \& Hamilton, A. L. (2013). Seeking qualitative rigor in inductive research: Notes on the Gioia methodology. Organizational research methods, 16(1), 15-31. DOI: $10.1177 / 1094428112452151$

Hall, D. J., \& Saias, M. A. (1980). Strategy follows structure!. Strategic management journal, 1(2), 149-163. DOI: $10.1002 / \mathrm{smj} .4250010205$

Hatch, M. J. with Cunliffe, A.(2006). Organization theory: Modern, symbolic and postmodern perspectives(2nd ed.). Oxford University Press.

Holmstrom, B., \& Roberts, J. (1998). The boundaries of the firm revisited. Journal of Economic perspectives, 12(4), 73-94.

Hope, O. L. E. (2010). The politics of middle management sensemaking and sensegiving. Journal of Change Management, 10(2), 195-215. DOI: 10.1080/14697011003795669

Isherwood, A., \& Tassabehji, R. (2016). A case analysis of managing "Maverick” innovation units. International Journal of Information Management, 36(5), 793-798.

DOI:10.1016/j.jijinfomgt.2016.05.014

Kapoor, R., \& Adner, R. (2012). What firms make vs. what they know: how firms' production and knowledge boundaries affect competitive advantage in the face of technological change. Organization Science, 23(5), 1227-1248. DOI: 10.1287/orsc.1110.0686

Kapoor, R., \& Klueter, T. (2015). Decoding the adaptability-rigidity puzzle: Evidence from pharmaceutical incumbents' pursuit of gene therapy and monoclonal antibodies. Academy of management journal, 58(4), 1180-1207. DOI: 10.5465/amj.2013.0430

Kapoor, R., \& Klueter, T. (2017). Organizing for New Technologies. MIT Sloan Management Review, 58(2), 85.

Kapoor, R., \& Lee, J. M. (2013). Coordinating and competing in ecosystems: How organizational forms shape new technology investments. Strategic management journal, 34(3), 274-296. DOI: 10.1002/smj.2010

Klueter, T., \& Monteiro, F. (2017). How does performance feedback affect boundary spanning in multinational corporations? Insights from technology scouts. Journal of Management Studies, 54(4), 483-510.

Liu, M., Denis, H., Kolodny, H., \& Stymne, B. (1990). Organization design for technological change. Human Relations, 43(1), 7-22. DOI: 10.1177/001872679004300102

Luke, R. D., Begun, J. W., \& Pointer, D. D. (1989). Quasi firms: Strategic interorganizational forms in the healthcare industry. Academy of Management Review, 14(1), 9-19. 
March, J. G., \& Simon, H. A. (1993). Organizations revisited. Industrial and Corporate Change, 2(3), 299-316.

Marengo, L., \& Pasquali, C. (2012). How to get what you want when you do not know what you want: A model of incentives, organizational structure, and learning. Organization Science, 23(5), 1298-1310.

McKinsey (2017). Smartening up with Artificial Intelligence (AI) - What's in it for Germany and its Industrial Sector?. Retrieved from

https://www.mckinsey.com/ /media/McKinsey/Industries/Semiconductors/Our\%20Insights/Smar tening\%20up\%20with\%20artificial\%20intelligence/Smartening-up-with-artificial-intelligence.as hx. [Accessed 2018-07-18].

McKinsey Global Institute (2017). What's now and next in Analytics, AI, and Automation. Retrieved from

https://www.mckinsey.com/ /media/mckinsey/featured\%20insights/digital\%20disruption/whats \%20now\%20and\%20next\%20in\%20analytics\%20automation/final\%20pdf/mgi-briefing-note-aut omation-final.ashx. [Accessed 2018-07-21].

McKinsey Global Institute (2018). How artificial intelligence and data add value to businesses. Retrieved from https://www.mckinsey.com/featured-insights/artificial-intelligence/how-artificial-intelligence-and -data-add-value-to-businesses. [Accessed 2018-07-22].

Miles, R. E., Snow, C. C., Meyer, A. D., \& Coleman Jr, H. J. (1978). Organizational strategy, structure, and process. Academy of management review, 3(3), 546-562.

Milgrom, P. R., \& Roberts, J. D. (1992). Economics, organization and management. Prentice-Hall International Inc.

Mintzberg, H. (1993). Structure in fives: Designing effective organizations. Prentice-Hall, Inc.

Patton, M. (2002). Qualitative research and evaluation methods (3rd ed.). Thousand Oaks, Calif. ; London: Sage Publications.

Pitts, R. A. (1980). The strategy-structure relationship: An exploration into causality. In National Academy of Management Meeting, Detroit.

Plastino, E., \& Purdy, M. (2018). Game changing value from Artificial Intelligence: eight strategies. Strategy \& Leadership, 46(1), 16-22. DOI: 10.1108/SL-11-2017-0106

Puranam, P. (2012). A Future for the Science of Organization Design. Journal of Organization Design, 1(1), 18-19. DOI: 10.7146/jod.6333

Puranam, P., Raveendran, M., \& Knudsen, T. (2012). Organization design: The epistemic interdependence perspective. Academy of Management Review, 37(3), 419-440.

Puranam, P., Alexy, O., \& Reitzig, M. (2014). What's “new” about new forms of organizing?. Academy of Management Review, 39(2), 162-180. DOI: 10.5465/amr.2011.0436

Puranam, P. (2017). When will we stop studying innovations in organizing, and start creating them?. Innovation, 19(1), 5-10.

Ransbotham, S., Kiron, D., Gerbert, P., \& Reeves, M. (2017). Reshaping Business With Artificial Intelligence: Closing the Gap Between Ambition and Action. MIT Sloan Management Review, 
59(1). Retrieved from

https://sloanreview.mit.edu/projects/reshaping-business-with-artificial-intelligence/. [Accessed 2018-07-20].

Ridder, H. (2012). Case Study Research. Design and Methods 4th ed. (Book Review). Zeitschrift Für Personalforschung / German Journal of Research in Human Resource Management, 26(1), 93-95.

Rivkin, J. W., \& Siggelkow, N. (2003). Balancing search and stability: Interdependencies among elements of organizational design. Management Science, 49(3), 290-311. DOI: 10.1287/mnsc.49.3.290.12740

Roberts, J. (2007). The modern firm: Organizational design for performance and growth. Oxford university press.

Siggelkow, N. (2011). Firms as systems of interdependent choices. Journal of Management Studies, 48(5), 1126-1140. DOI: 10.1111/j.1467-6486.2011.01010.x

Stebbins, R. A. (2001). Exploratory research in the social sciences (Vol. 48). Sage Publications Inc. DOI: $10.4135 / 9781412984249$

Teachey, (n.d.). Data governance framework: What is it and do I already have one?. Retrieved from https://www.sas.com/en_gb/insights/articles/data-management/what-is-a-data-governance-framewor k.html. [Accessed 2018-07-20].

Thompson, J. D. (1967). Organizations in action. New York, McGraw-Hill.

Tushman, M., Lakhani, K., \& Lifshitz-Assaf, Hila.(2012). Rethinking Organization Design for Complex Endeavours. Journal of Organization Design, 1(1), 24-27. DOI: 10.7146/jod.6333

Von Krogh, G. (2018). Artificial Intelligence in Organizations: New Opportunities for Phenomenon-Based Theorizing. Academy of Management Discoveries. DOI: 10.5465/amd.2018.0084

Wise, L. (2016). Understanding data governance. Retrieved from https://www.cio.com/article/3116812/business-intelligence/understanding-data-governance.html. [Accessed 2018-07-22].

Yin, R. K. (2012). A (very) brief refresher on the case study method. Applications of case study research, Sage Publishers, pp.3-20.

Yin, R. K. (2014). Case study research: Design and methods (Fifth). Thousand Oaks, California, SAGE 
Institute for Policy Analysis and Social Innovation, University of Hyogo

Graduate School of Disaster Resilience and Governance, University of Hyogo

Published online: May, 14, 2019

Japan Social Innovation Journal, Vol. 8, No. 1, 2019

Appendix I:

Interviewee profile

\begin{tabular}{|l|l|l|l|}
\hline Code & Tenure of service & Position/Role & Gender \\
\hline P1 & 4.5 years & $\begin{array}{l}\text { Head of Technology, X technology } \\
\text { team }\end{array}$ & Male \\
\hline P2 & 2.5 years & $\begin{array}{l}\text { Software Engineer, X technology } \\
\text { team }\end{array}$ & Male \\
\hline P3 & 16 years & General Manager & Male \\
\hline P4 & 17 years & Head of Platform Services & Male \\
\hline P5 & 12 years & General Manager & Male \\
\hline P6 & 16 years & CoO/General Manager & Female \\
\hline P7 & 8 years & Simulation Development Manager & Male \\
\hline P8 & 8 years & $\begin{array}{l}\text { Head of Robotics and Autonomous } \\
\text { Systems }\end{array}$ & Male \\
\hline P9 & 9 years & Development Manager & Male \\
\hline P10 & 13 years & $\begin{array}{l}\text { Development Manager, X } \\
\text { technology team }\end{array}$ & Male \\
\hline P11 & 3 years & Data Science Team Leader & Female \\
\hline P12 & 12 years & Chief Technology Officer (Group) & Male \\
\hline P13 & 9 years & Head of Department & Male \\
\hline P14 & 8 years & Data Science Team Leader & Male \\
\hline P15 & 7.5 years & $\begin{array}{l}\text { Chief Technology Officer }<\text { Product } \\
\text { segment> }\end{array}$ & Male \\
\hline
\end{tabular}

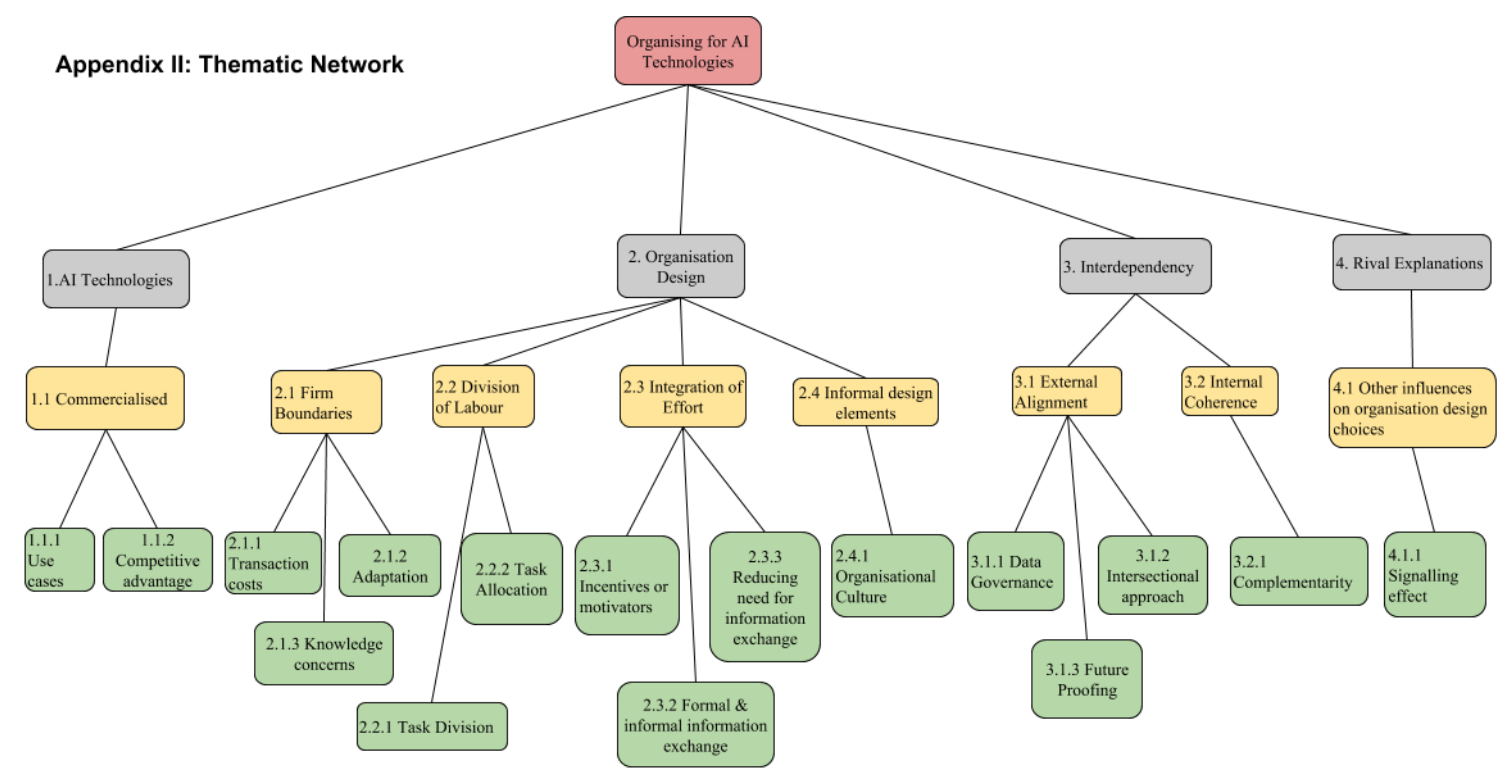


Institute for Policy Analysis and Social Innovation, University of Hyogo

Graduate School of Disaster Resilience and Governance, University of Hyogo

Published online: May, 14, 2019

Japan Social Innovation Journal, Vol. 8, No. 1, 2019
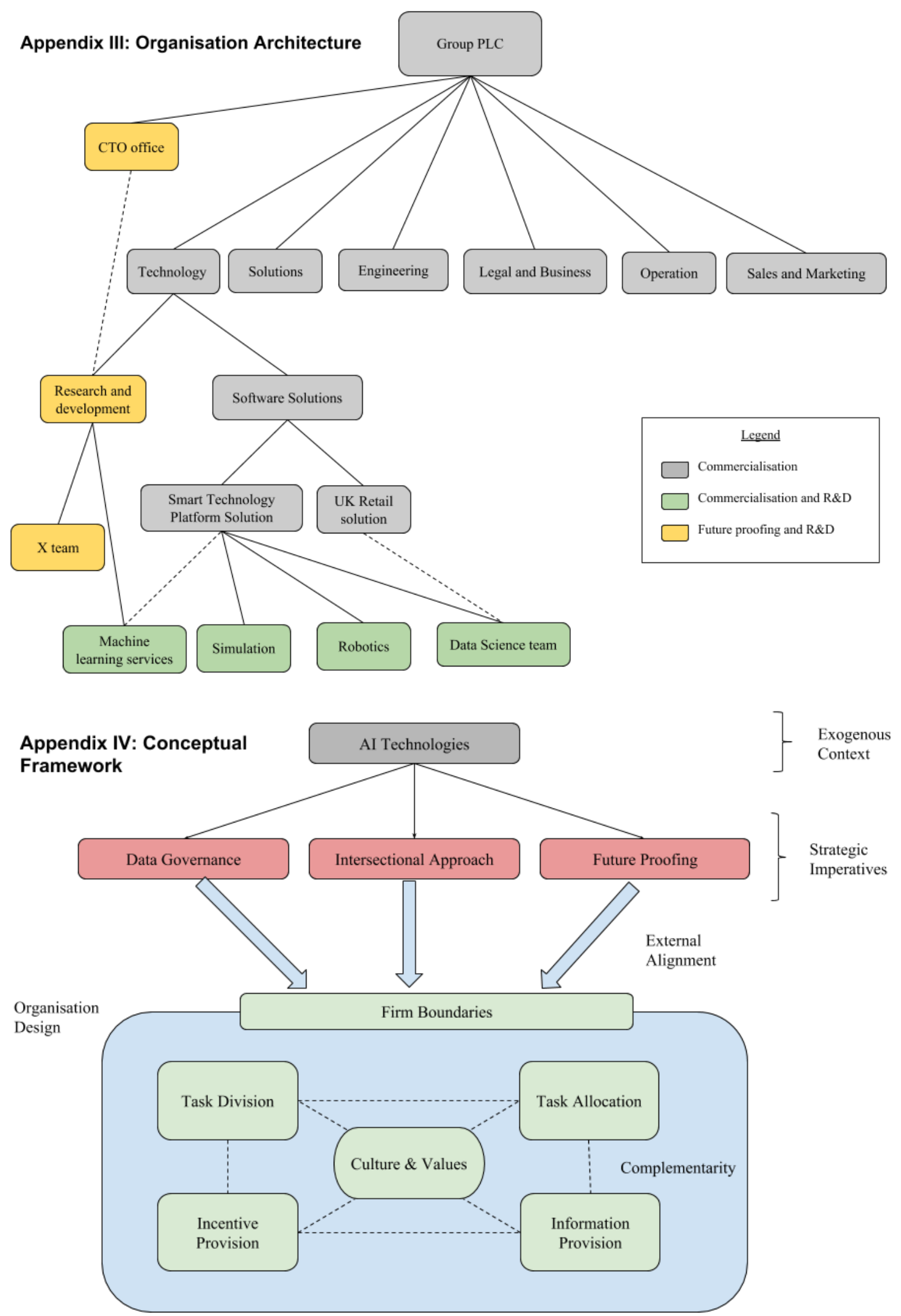